\title{
1 Blood Loss in Total Knee Arthroplasty
}

3 Background

4 Patients undergoing Total Knee Arthroplasty (TKA) have expected blood loss during and after

5 surgery. The morbidity associated with blood loss and the burden of blood transfusions in adult

6 arthroplasty necessitates pre-operative optimisation as routine practice. Current literature remains

7 inconclusive on which TKA surgical instrumentation techniques are effective in minimising peri-

8 operative blood loss, and consequently lower transfusion rates. The primary objective of this

9 retrospective review, of a prospective randomized cohort study, was to compare surgical and patient

10 factors, and their influence on blood loss and transfusions rates, between one type of Patient Specific

11 Instrumentation (PSI), Navigated Computer-Assisted Surgery (CAS) and Conventional TKA

12 surgical techniques.

14 Methods

15 A cohort of 128 matched patients (38 PSI, 44 CAS, 46 Conventional surgeries) were compared. Pre-

16 operative factors analysed included; age, gender, Body Mass Index (BMI), pre-operative hemoglobin

$17(\mathrm{Hb})(\mathrm{g} / \mathrm{L})$, International Normalized Ratio (INR), use of anticoagulants and co-morbid bleeding

18 diathesis. Maximal $\mathrm{Hb}$ drop and transfusion requirements were compared on Day 1 to 3. Peri-

19 operative factors collected included; surgical time, tourniquet time, drain output, insitu drain time,

20 order of tibia or femoral cut and intra-operative loss from suction.

Results

23 The 3 groups did not differ on the pre-operative patient demographics examined. The difference

24 between pre-operative $\mathrm{Hb}$ and the lowest post-operative $\mathrm{Hb}$ readings did not differ between study

25 groups $(\mathrm{p}=0.39)$. 


\section{Conclusion}

28 There are no statistically significant differences in blood loss when comparing PSI vs. CAS vs.

29 Conventional TKA. Although emerging evidence on PSI is encouraging, the PSI technique for TKA

30 does not result in reduced blood loss.

31

32 Registration: ClinicalTrials.gov: NCT01145157

33 Key Words: Blood Loss; Surgical Technique; Total Knee Arthroplasty; Patient Specific

34 Instrumentation (PSI); Transfusion. 


\section{1. Introduction}

38 Peri-operative blood loss and the requirement for blood product transfusion after joint replacement

39 have been extensively studied. Total Knee Arthroplasty (TKA) results in substantial blood loss and

40 patients undergoing total joint replacement are at increased risk for transfusion. Following TKA,

41 transfusion rates have been reported as high as 39\%..$^{1}$ There has been a paradigm shift to reduce the

42 need for post-operative transfusion by improving peri-operative blood management and positively

43 impact early and long-term outcomes.

45 The current literature includes many studies assessing the potential benefit of navigated Computer-

46 Assisted Surgery (CAS) TKA against Conventional TKA, producing mixed findings. CAS TKA was

47 proposed to allow reduced blood loss due to the avoidance of the medullary cavity. ${ }^{2}$ Findings from

48 previous studies support this concept, reporting reductions in blood loss, lower Hemoglobin $(\mathrm{Hb})$

49 drop and the risk of post-operative transfusion. ${ }^{2,3}$ Schnurr et al. (2010) also reported reduced blood

50 loss in patients implanted via CAS, resulting in a 50.0\% reduction in transfusion rate in comparison

51 to patients having received Conventional TKA. ${ }^{4}$ Similarly, findings from a randomized controlled

52 trial reported less drained blood, however no statistically significant difference in average Hb drop

53 and allogenic transfusion rate. ${ }^{5}$

55 Recent literature has demonstrated no significant difference in blood loss ${ }^{6}$, post-operative Hb or the 56 need for transfusion between Conventional and CAS TKA. ${ }^{7,8}$ Similarly to CAS TKA, Patient

57 Specific Instrumentation (PSI) assisted TKA does not breach the medullary canals, potentially

58 resulting in reduced blood loss and a lower risk of transfusion. ${ }^{9}$ A comprehensive search of the

59 literature produced one study that considered PSI and the effect on blood loss. Theinpont et al. 
60 (2014) reported no statistically significant difference between calculated blood loss, Hb drop and

61 transfusion rate between PSI-assisted and conventional minimally invasive surgical techniques. ${ }^{9}$

62

63 Current literature examines CAS vs Conventional and PSI vs Conventional, but no paper exists

64 comparing all 3 techniques in 1 matched cohort. This retrospective review of a prospective

65 randomized cohort aims to compare these 3 surgical techniques (PSI, CAS, and Conventional TKA)

66 to determine which technique, if any, results in reduced blood loss and therefore a lower risk of

67 transfusion. Our hypothesis was that use of the PSI technique would lead to reduced blood loss and

68 post-operative $\mathrm{Hb}$ drop. 


\section{2. Materials and Methods}

72 This retrospective review was approved by the Southern Adelaide Clinical Human Research Ethics

73 Committee (SAC HREC 300.14, June 2014) and the prospective cohort study was registered on a

74 Clinical Trials registry (ClinicalTrials.gov: NCT01145157).

2.1 Study Selection

All TKA patients listed on our institution's routine elective arthroplasty clinic were screened for study eligibility. Patients meeting study selection criteria were invited to participate in the trial.

\subsubsection{Inclusion criteria}

82 Patients were of legal age and skeletal maturity, and required primary TKA due to non-inflammatory

83 degenerative joint disease (e.g. osteoarthritis, traumatic arthritis, avascular necrosis, and

84 dysplasia/DDH) or inflammatory joint disease (e.g. Rheumatoid Arthritis). Patients met an

85 acceptable pre-operative medical clearance and were free from, or treated for, cardiac, pulmonary,

86 hematological or similar conditions that posed excessive operative risk. Patients were required to be

87 willing and able to provide written informed consent to participate in the length of the study.

\section{$89 \quad 2.1 .2 \quad$ Exclusion criteria}

90 Patients were excluded from study participation if they had history of active infection or sepsis

91 (treated or untreated), vascular insufficiency, muscular atrophy, or neuromuscular disease at a

92 severity to compromise implant stability or post-operative recovery. Restrictions were also placed for

93 patients with inadequate bone stock to support the device (e.g., severe osteopenia or family history of 
94 severe osteoporosis), known moderate to severe renal insufficiency or metal sensitivity, a history of

95 previous knee surgery (except arthroscopy and/or open meniscectomy) on the affected knee, or a

96 Body Mass Index (BMI) > 40. Further exclusions were immunocompromised patients or those

97 receiving high doses of corticosteroids, as well as patients with an emotional or neurological

98 condition that pre-empted their ability or willingness to participate in the study, including mental

99 illness, intellectual disability, drug or alcohol abuse or a contraindication to MRI (metal artifacts in

100 soft tissue, claustrophobia, body mass). Female patients of child-bearing age not taking contraceptive

101 precautions were ineligible for study participation.

103 Eligible patients were consented by research staff following a comprehensive explanation of the

104 study process. Participants were then randomized into 1 of the 3 study arms, PSI, CAS or

105 Conventional TKA. Information relating to functional scores, radiographic evaluations, operative

106 details, discharge details, end of study and missed/unplanned visits were captured. A 1:1:1 block

107 randomization was used in the study. The assignments were predetermined through a schedule

108 generated using randomly permuted blocks with random block sizes method. Randomization plan

109 generator available on the randomization.com website was used. During post-operative reviews,

110 radiologists were blinded to the assignments until individual randomization assignments were

111 requested.

113 A total of 5 consultant Orthopedic Surgeons were the primary surgeons for this cohort. All surgeons

114 were experienced with the 3 techniques and underwent industry training for the Signature ${ }^{\mathrm{TM}}$ system.

115 Patients randomized to the PSI Personalised Patient Care arm had an MRI 6 weeks pre-operatively.

116 The MRI image was used to build surgical instruments customised for a patient's unique knee

117 anatomy. Cutting positioning guides were produced to match the outer shape of the individual's 
118 distal femur and proximal tibia. Consultants on the study team reviewed the pre-operative plans

119 before the final guides were manufactured.

121 This was a single centre study with 150 patient enrolled. A number of participants (22) were 122 excluded due to the following reasons; inability to undergo pre-operative MRI, voluntary

123 withdrawal, PSI template molds not available at time of surgery and computer-assisted navigation 124 abandoned due to software/instrument issue. A number of participants were withdrawn from the PSI 125 group (13) and the CAS group (9) as they were converted to undergo Conventional TKA. Therefore, 126 in this study, 128 patients were available for comparison comprising the PSI (Signature, Biomet, 127 Warsaw, USA), CAS and Conventional TKA, using the Vanguard Knee System. A total of 38 128 patients were randomized to PSI, 44 to CAS and 46 to Conventional implants.

\subsection{Pre-Operative factors}

132 Pre-operative factors were collected to assess homogeneity between the 3 groups. Baseline 133 demographics included (Table 1); age, gender, BMI, pre-operative $\mathrm{Hb}(\mathrm{g} / \mathrm{L})$, International 134 Normalized Ratio (INR), use of anticoagulants, determining if held prior to surgery and co-morbid 135 bleeding diathesis. Routinely, all medications that had an influence on bleeding were ceased 136 (including nutraceuticals i.e. Fish Oil). Exceptions were Aspirin or Clopidogrel, which were 137 continued in cases of ischemic heart, or valvular disease as directed by Peri-Operative Physicians.

138 Complete blood count, biochemistry and coagulation bloods tests were performed as routine pre139 operatively. 
143 Length of surgical time, tourniquet time, order of tibia or femoral cut and intra-operative loss from

144 suction including estimated blood loss from weighed packs were collected at time of surgery.

1462.4 Post-operative factors

148 At the study institution it is standard practice for all patients to have a blood sample taken on post149 operative Day 1. Thereafter, blood sampling is determined on an individual patient basis depending

150 on their clinical circumstances. The minimum and maximum post-operative time that elapsed before 151 a valid $\mathrm{Hb}$ measurement was taken and included for analysis was 6 hours and 72 hours, respectively.

152 The protocol for the study institution was to transfuse if $\mathrm{Hb}<80$ or if the patient is symptomatic of 153 anemia (e.g. tachycardia or hypotensive). It is presumed study patients did not receive tranexamic 154 acid, as the use of this drug was not standard practice in the department at the time in which the 155 study was conducted.

157 The thromboprophylaxis protocol at study institution standardizes mechanical prophylaxis for all 158 patients in the form of compression stockings and sequential pneumatic pumps. Pharmacological 159 prophylaxis is stratified according to individual patient risk profile. High-risk patients (usually on 160 warfarin pre-operatively) are commenced on bridging enoxaparin and warfarin post-operatively.

161 Enoxaparin is ceased when INR values stabilize in the therapeutic range and then warfarin is 162 continued for 3 months duration. Low-risk patients are commenced on Aspirin 150mg daily for 6 163 weeks post-operatively.

165 The use of a drain was determined by surgeon preference. In patients with drains, the total output at 166 time of removal was recorded in medical records and fluid balance charts by nursing or medical 167 staff. 
1692.5 Statistical analysis

170

171 All analyses were conducted with IBM SPSS Statistical software package. Differences in age,

172 gender, BMI, tourniquet time, pre-operative INR, pre-operative anticoagulation medication, between

173 groups were determined using the Chi-squared or Fisher's exact test. Additionally, differences in

174 requirement for wound drains and post-operative drain insertion time were compared between

175 groups using the Kruskall-Wallis test. For all association tests, a P-value ( $p$ ) of less than 0.05 was

176 considered statistically significant. 


\section{Results}

179 A total of 128 patients comprised the study cohort, representative of 44 CAS, 46 conventional and 38

180 PSI. Demographic information is summarised in Table 1 and displays no significant difference in

181 age, gender or BMI between the 3 groups. A significant difference in tourniquet time was identified,

182 and routine pre-operative baseline blood tests demonstrated comparable pre-operative Hb levels

183 across the 3 groups $(\mathrm{p}=0.23)$.

185 Pre-surgery factors appeared comparable, with no significant differences seen between the 3 groups.

186 Patient demographics that were matched included; age, BMI, pre-operative $\mathrm{Hb}$, INR and

187 anticoagulant use.

3.1 Anticoagulant use/ holding pre-operatively

191 A total of 16 patients had raised INR pre-operatively and were spread as follows; 4 (PSI group), 4

192 (CAS group) and 8 (Conventional group). In the PSI group, 2 of the 4 patients were on warfarin 193 which was held pre-operatively for 7 days. INR results for these 2 patients were 1.1 and 1.4. The 194 remaining 2 patients were not on warfarin and both had an INR of 1.1.

196 No patients in the CAS group were taking warfarin and all had an INR of <1.2. The 4 patients with

197 INR of 1.1 or 1.2 are not representative of a higher bleeding potential. INR was developed to

198 specifically monitor warfarin therapy and may not be used as an assay for bleeding potential in 199 patients not taking warfarin ${ }^{10}$. In the Conventional group, 2 of the 8 patients were on warfarin and 200 this was held pre-operatively for 7 days. INR results were 1.2 and 1.3, respectively. The remaining 6 
201 patients were not on warfarin and their INR levels were $\leq 1.2$. The study authors did not consider

202 raised INR levels on non-warfarin patients to be a contributing factor to bleeding risk.

204 The PSI group had a single patient who was continued on Celebrex despite instructions. However, 205 this patient was not excluded as this was discovered post-operatively, and blood loss in this patient 206 was within normal range. All anticoagulants were withheld in the CAS group. A total of 3 patients 207 were continued on Aspirin in the Conventional group due to cardiac or valvular co-morbidities.

2093.2 Surgical Factors

211 Surgical factors are summarised in Table 2. A significant difference was found between surgical 212 times across the 3 groups, with the CAS group displaying the longest surgical time (Fig. 1). The 213 difference in tourniquet time between the 3 groups was also significant (Fig. 1). The CAS group had 214 the longest time with a mean of 78 minutes in contrast to the shortest mean time of 61 minutes in the 215 PSI group. The tourniquet was routinely inflated prior to first skin incision in all cases except 5

216 cases. The 5 cases used short tourniquet time for the cementing process only and cases were evenly 217 spread across the three groups (Conventional (2), CAS (2) and PSI (1)). Length of surgery over time 218 for the PSI group is displayed in Fig. 2.

220 A significant association was observed between use of drains and surgical treatment group. The 221 frequency of drain use appeared greater in the Conventional group (54.35\%) compared to the other 2 222 groups. The use of drains was due to surgeon preference. The difference in Length of Stay in 223 Hospital (LOS) was not significant, with a mean LOS of 5 days across all 3 groups. No significant 224 association was observed between groups and whether the tibia or femoral component was cut and 225 implanted first. 
227 The difference in intra-operative blood loss between the 3 groups was also not significant. The 228 observed blood loss was recorded at the time of surgery, with PSI and Conventional both recording a 229 mean observed loss of $100 \mathrm{ml}$ while CAS recorded $150 \mathrm{ml}$.

231 As displayed in Table 3, no significant difference between the mean pre- and post-op Hb levels was 232 present between the 3 groups. However, a significant difference was observed with respect to Day 1 $233 \mathrm{Hb}$. The difference between Pre-Op $\mathrm{Hb}$ and the lowest of the Post-Op Hb readings did not differ 234 between the 3 groups (Fig. 3). In this study, 4 patients required transfusion due to either $\mathrm{Hb}<80 \mathrm{~g} / \mathrm{l}$ or 235 clinically symptomatic. These 4 patients were all from the Conventional TKR group.

237 Drains were used in 3 of the 4 cases in the Conventional group that required transfusion. Total drain 238 output for these patients were $550 \mathrm{ml}, 700 \mathrm{ml}$ and $1000 \mathrm{ml}$, with time in situ for these drains being 24 239 hours, 20 hours and 28 hours, respectively.

241 The study captured a 3-day post-operative period in which two complications were reported. One 242 patient experienced a dislocation of the operative knee, while the second had a pulmonary embolism 2432 days post TKA. Both patients were in the PSI group and complications were resolved. In an 244 extended review of complications reported as part of the prospective cohort study an additional 5 245 significant surgical, bleeding and thromboembolic complications were identified. A further 2 patients 246 from the PSI group reported complications including a wound haematoma 10 days post-operatively 247 and a manipulation under anesthesia procedure. Complications reported from patients in the 248 conventional group included a pulmonary embolism 19 days post-operatively and a knee 249 haemarthrosis. A single patient from the CAS group experienced failure of the polyethylene device 
250 locking clip which was resolved via revision of the polyethylene component.

251

252

253 


\section{Discussion}

255

256 This study sought to investigate blood loss between 3 surgical techniques for TKA. The PSI and

257 CAS groups do not perforate the intramedullary cavity ${ }^{9,11}$ and thereby reducing intra-operative 258 systemic emboli. ${ }^{12}$ The current literature is inconclusive on if the PSI or navigation offer increased 259 accuracy in component placement. ${ }^{9,11}$ Reduced complications, Length of Stay (LOS) and transfusion 260 requirements through a more accurate and less disruptive method is desirable. However, in this study

261 the most important finding was no significant difference between blood loss across the 3 groups.

263 Across all 3 study groups BMI ranged from 21.2 to 44.1 with an average of 31 . A BMI of 31 is 264 classified as 'Moderately Obese' according to the WHO criteria. ${ }^{13}$ In a recent systematic review of 265 morbidity and TKR, complications were significantly higher in morbidly obese patients $(\mathrm{BMI}<40)$.

266 Complications higher in the BMI > 40 group included superficial and deep infections, deep vein 267 thrombosis and avulsion of the medial collateral ligament. ${ }^{14}$

269 The sugical time was signficantly different between the 3 groups. The navigation group had the 270 highest average surgical time $(110 \mathrm{~min})$ and longest mean tourniquet time. Longer tourniquet time 271 and total surgical time has been reported previously in the literature for patients undergoing CAS 272 knee arthroplasty. ${ }^{2,15,16}$ Increased tourniquet time is associated with increased wound infection, tissue 273 ischemia, DVT and anesthetic risk. ${ }^{15}$ Rama et al. (2007) found that early tourniquet release increased 274 both the calculated blood loss and the total measured blood loss but not the post-operative blood 275 loss. ${ }^{17}$ This indicates that early release of tourniquet may contribute to increased total blood loss at 276 the intra-operative stage. ${ }^{17}$ In this study, the timing of release of tourniquet was at the surgeon's 277 preference and not routinely deflated before or after wound closure. The use of diathermy to ensure 278 haemostasis prior to wound closure was anecdotally the most frequently used technique, however 
279 some surgeons may have relied on local compressive or tamponade effects to stem bleeding when 280 releasing tourniquet after wound closure. The risk of a complication requiring additional surgery is

281 significantly increased when the tourniquet was left inflated until wound closure was complete. ${ }^{17}$

282 The data on timing of tourniquet release was not recorded prospectively, or available retrospectively.

283 This is a limitation of this study, as release of tourniquet can occur at any stage of the operation at

284 surgeon's discretion. Our data suggests that the intra-operative blood loss observed from suction was 285 not significantly different between the 3 groups, hence the timing of tourniquet release was not 286 considered a major factor in recording post-operative losses.

288 The learning curve associated with the new Signature ${ }^{\mathrm{TM}}$ PSI system was evaluated using the surgical 289 length of time as a surrogate marker of experience and skill with the system. Figure 2. depicts a 290 varying surgical time over the 4 year study period and no definite trend line exists in this group to 291 suggest decrease in surgical time over the study period.

293 The use of drains in TKA is controversial. Literature supports no statistically significant difference in 294 post-operative $\mathrm{Hb}$ levels or allogenic blood transfusion requirement between patients with no drain, 295 closed drain or reinfusion drain. ${ }^{18,19}$ In our study a drain was used for $33 \%$ in PSI, $15 \%$ in CAS and $29654 \%$ in the Conventional group. This difference in drain use was statistically significant, however, 297 literature supports the authors' view that drain use does not represent a confounding factor in our 298 results. A limitation of this study was that the recordings of drain time removal and total blood 299 collected at time of removal was reliant on recording of fluid balance chart/documentation in nursing 300 notes in medical records. The element of human error and lack of documentation is apparent in 301 retrospective chart reviews. ${ }^{20}$ 
303 Mean LOS in hospital was 5 days across the 3 study groups. LOS is important for reduction of

304 healthcare costs, hospital efficiency and management of patient expectations. ${ }^{21}$ The use of hospital

305 stay time as a surrogate marker for post-operative recovery and hospital efficiency has seen the mean

306 LOS decrease from 7.9 days in 1991 to 3.5 days in $2010 .^{22}$ There was no significant difference

307 between LOS across the 3 arthroplasty groups in our study, reflecting similar co-morbidities and

308 adequate assistance at home.

310 Blood loss in total knee replacement is a well published entity and a known complication in

311 arthroplasty. Calculations have been used in previous studies to assess factors such as hemodilation

312 from crystalloids and blood volume to determine a cumulative blood loss. ${ }^{23,24}$ This study sought to

313 assess each variable individually. The testing of hematocrit was not routinely performed after day 1

314 post - surgery, as compared with other studies, which relied on hematocrit in combination with $\mathrm{Hb}$

315 to determine blood loss.

316 We followed the paper by Thienpont et al. ${ }^{9}$ in their method comparison of pre-operative Hb and

317 lowest post-operative to gauge the maximal drop. Measured blood loss after TKA is generally an

318 underestimation and can be only $50 \%$ of the "true" blood loss, as calculated with several

319 methods. $^{25,26}$ This finding suggests that the available methods of measuring intra-operative blood 320 loss are inefficient.

321 The mean $\mathrm{Hb}$ pre-operative was 140 for PSI, 135 for CAS and 139 for conventional. The mean post322 operative $\mathrm{Hb}$ was 111 for PSI, 104 for CAS and 107 for conventional. Thereby calculating that the 323 mean percentage drop was $21 \%, 23 \%$ and $23 \%$, respectively. No significant difference was found 324 between mean pre- and post-operative $\mathrm{Hb}$ levels.

325 The difference between pre-op and Day 1 post-op Hb levels was not significant $(\mathrm{p}=0.05)$. The 326 timing of day 1 levels was not uniform. In some patients the Day 1 blood test occurred in the 
327 afternoon on same day of surgery, for others it was the following morning. In addition, the

328 hematocrit was not taken to assess for hemodilation as a contributing factor.

329 The authors prefer pre-op and lowest post-op as a method of analysing Hb drop data. The lowest post

330 - operative sample may have occurred on Day 1, Day 2 or Day 3. The latter 2 would have allowed

331 for fluid balance homeostasis, however, further blood tests were at the discretion of the treating team

332 and not routinely performed.

333

334 Despite these limitations, Anesthesia and post-operative care were standard across all groups in the

335 same conditions. DVT prophylaxis protocol was uniformly used in all patients and no patients were

336 found to have DVT and commenced on therapeutic anticoagulation during first 3 days post-surgery.

337 The decision to transfuse or not was deemed in accordance with the institution protocol ( $\mathrm{Hb}<80$ or

338 symptomatic of anemia).

339

340 The strength of this study is that blood values were obtained at regular post-operative times with

341 daily phlebotomy rounds. In addition, pre-operative pharmacist and anesthetic consultations

342 documented the recordings of anticoagulant use. The patient demographics and accountability of

343 randomization was also a strength.

345 The 4 patients who required transfusion from the Conventional group all recorded Hb levels below

346 90. Of these, 1 patient had a recorded 1 liter intra-operative blood loss and was transfused in the

347 immediate post-operative period.

348

349 5. Conclusions

350 It was hypothesized that performing TKA surgery using PSI technology would lead to lower intra-

351 operative blood loss and a reduced drop in post-operative $\mathrm{Hb}$. The technique does not require the use 
352 of intramedullary alignment rods and the soft tissue dissection is less extensive than in the other 353 techniques.

354

355 Our results demonstrate that surgical technique did not influence post-operative blood loss when 356 comparing PSI, CAS and Conventional TKA. This is the first article to compare all 3 knee

357 arthroplasty systems in a matched patient series. The surgeon's preference of drain use did not 358 appear to influence post-operative blood loss.

359

360

361

362

363

364

365

366

367

368

369

370

371

372

373

374

375

376 
379 1. Callaghan JJ, Spitzer AI. Blood management and patient specific transfusion options in total 380 joint replacement surgery. The Iowa orthopaedic journal. 2000;20:36-45.

381 2. Kalairajah Y, Simpson D, Cossey AJ, Verrall GM, Spriggins AJ. Blood loss after total knee 382 replacement: effects of computer-assisted surgery. The Journal of bone and joint surgery. British volume. 2005;87(11):1480-1482.

384 3. Conteduca F, Massai F, Iorio R, Zanzotto E, Luzon D, Ferretti A. Blood loss in computer385 assisted mobile bearing total knee arthroplasty. A comparison of computer-assisted surgery with a conventional technique. International orthopaedics. 2009;33(6):1609-1613.

4. Schnurr C, Csecsei G, Eysel P, Konig DP. The effect of computer navigation on blood loss and transfusion rate in TKA. Orthopedics. 2010;33(7):474.

5. Hinarejos P, Corrales M, Matamalas A, Bisbe E, Caceres E. Computer-assisted surgery can reduce blood loss after total knee arthroplasty. Knee surgery, sports traumatology, arthroscopy : official journal of the ESSKA. 2009;17(4):356-360.

6. Chang CW, Wu PT, Yang CY. Blood loss after minimally invasive total knee arthroplasty: effects of imageless navigation. The Kaohsiung journal of medical sciences. 2010;26(5):237-

395 7. Graham DJ, Harvie P, Sloan K, Beaver RJ. Morbidity of navigated vs conventional total knee arthroplasty: a retrospective review of 327 cases. The Journal of arthroplasty. 2011;26(8):1224-1227.

398 8. Mohanlal PK, Sandiford N, Skinner JA, Samsani S. Comparision of blood loss between 399 computer assisted and conventional total knee arthroplasty. Indian journal of orthopaedics. 2013;47(1):63-66. 
4019 9. Thienpont E, Grosu I, Paternostre F, Schwab PE, Yombi JC. The use of patient-specific 402 instruments does not reduce blood loss during minimally invasive total knee arthroplasty? 403 Knee surgery, sports traumatology, arthroscopy : official journal of the ESSKA. 2014.

404 10. Hirsh J, Poller L. The international normalized ratio. A guide to understanding and correcting 405 its problems. Arch Intern Med. 1994;154(3):282-288.

406 11. Bauwens K, Matthes G, Wich M, et al. Navigated total knee replacement. A meta-analysis. 407 The Journal of bone and joint surgery. American volume. 2007;89(2):261-269.

408 12. Kalairajah Y, Cossey AJ, Verrall GM, Ludbrook G, Spriggins AJ. Are systemic emboli 409 reduced in computer-assisted knee surgery?: A prospective, randomised, clinical trial. The Journal of bone and joint surgery. British volume. 2006;88(2):198-202.

411 13. Thienpont E, Grosu I, Jonckheere S, Yombi JC. C-reactive protein (CRP) in different types of minimally invasive knee arthroplasty. Knee surgery, sports traumatology, arthroscopy : official journal of the ESSKA. 2013;21(11):2603-2610.

414 14. Samson AJ, Mercer GE, Campbell DG. Total knee replacement in the morbidly obese: a 415

416 15. Ajwani SH, Jones M, Jarratt JW, Shepard GJ, Ryan WG. Computer assisted versus 417 conventional total knee replacement: a comparison of tourniquet time, blood loss and length $418 \quad$ of stay. The Knee. 2012;19(5):606-610.

419 16. Luring C, Bathis H, Tingart M, Perlick L, Grifka J. Computer assistance in total knee 420 replacement - a critical assessment of current health care technology. Computer aided surgery : official journal of the International Society for Computer Aided Surgery. 2006;11(2):77-80.

423 17. Rama KR, Apsingi S, Poovali S, Jetti A. Timing of tourniquet release in knee arthroplasty. 424 Meta-analysis of randomized, controlled trials. The Journal of bone and joint surgery. American volume. 2007;89(4):699-705. 
426 18. Adalberth G, Bystrom S, Kolstad K, Mallmin H, Milbrink J. Postoperative drainage of knee arthroplasty is not necessary: a randomized study of 90 patients. Acta orthopaedica Scandinavica. 1998;69(5):475-478.

19. Al-Zahid S, Davies AP. Closed suction drains, reinfusion drains or no drains in primary total knee replacement? Annals of the Royal College of Surgeons of England. 2012;94(5):347-350.

20. Braaf S, Manias E, Riley R. The role of documents and documentation in communication failure across the perioperative pathway. A literature review. International journal of nursing studies. 2011;48(8):1024-1038.

434 21. Halawi MJ, Vovos TJ, Green CL, Wellman SS, Attarian DE, Bolognesi MP. Preoperative Predictors of Extended Hospital Length of Stay Following Total Knee Arthroplasty. The Journal of arthroplasty. 2014.

22. Cram P, Lu X, Kates SL, Singh JA, Li Y, Wolf BR. Total knee arthroplasty volume, utilization, and outcomes among Medicare beneficiaries, 1991-2010. Jama. 2012;308(12):1227-1236.

24. Meunier A, Petersson A, Good L, Berlin G. Validation of a haemoglobin dilution method for estimation of blood loss. Vox sanguinis. 2008;95(2):120-124.

444 25. Gross JB. Estimating allowable blood loss: corrected for dilution. Anesthesiology. 1983;58(3):277-280.

446 26. Sehat KR, Evans R, Newman JH. How much blood is really lost in total knee arthroplasty?.

447 Correct blood loss management should take hidden loss into account. The Knee.

$448 \quad$ 2000;7(3):151-155.


451 Figures:

452

453 Figure 1. Mean Surgical and Tourniquet Time

454 Blue Columns: Mean Surgical Time (minutes) per surgical technique

455 Green Columns: Mean Tourniquet time (minutes) per surgical technique

456

457 Figure 2. Surgical time over the study period for the Patient - Specific Instrumentation (PSI) Group 458

459 Figure 3. Hemoglobin Drop - Mean Pre - Op, Post - Op and Maximal Hb Drop

460 Error Bars: 95\% Confidence Interval (CI)

461 Blue Columns: Pre-operative $\mathrm{Hb}$

462 Green Columns: Post-operative $\mathrm{Hb}$

463 Grey Columns: Peri-operative Differential $\mathrm{Hb}^{*}$ 